\author{
Stefan Lönnerholm, Helena \\ Malmborg, Per Blomström and Carina \\ Blomström-Lundqvist \\ Department of Medical Sciences, Department of \\ Cardiology, Uppsala University, Sweden
}

Dates: Received: June 17, 2014; Accepted: July 05, 2014; Published: July 07, 2014

*Corresponding author: Stefan Lönnerholm, MD, Institution of Medical Sciences, Department of Cardiology, Uppsala University, SE - 75185 Uppsala, Sweden, Tel: +46 1861100 00; Fax: +46 18 510243; E-mail: stefan.Ionnerholm@akademiska.se

www.peertechz.com

Keywords: Atrial fibrillation; Ablation; Radiofrequency energy; Duty-cycled; PVAC

ISSN: 2455-2976

\section{Research Article \\ Efficacy and Safety of Different Energy Settings for Atrial Fibrillation Ablation using the Duty-cycled Radiofrequency Ablation Catheter (PVAC)}

\begin{abstract}
In recent years, new ablation catheters have been developed to facilitate atrial fibrillation $(A F)$ ablation procedures, including the multi-electrode pulmonary vein ablation catheter (PVAC), capable of using different proportions of unipolar and bipolar energy. The purpose of this study was to evaluate the acute efficacy, as well as safety, when using the two different energy settings for the PVAC catheter

Methods and Results: Thirty-five patients with a mean age of $62 \pm 7.7$ years with paroxysmal $(54 \%)$ or persistent AF were included in the study and randomized to $4: 1$ versus 2:1 bipolar/unipolar energy setting with the PVAC device. The mean number of applications with the PVAC catheter was $41 \pm 10$ and $51 \pm 15(p=0.3)$ respectively with the $4: 1$ and the $2: 1$ setting. Touch-up with another RF ablation catheter was necessary in 3 and 7 patients respectively in the $4: 1$ and $2: 1$ group. The procedure time was $155 \pm$ 35 and $174 \pm 41$ minutes respectively and the total fluoroscopy time, including the time for touch-up with another catheter, was $42 \pm 14$ and $50 \pm 17$ minutes respectively with the $4: 1$ versus 2:1 setting. No complications were seen in any group.

Conclusion: There was no significant difference between the $4: 1$ and 2:1 bipolar-tounipolar energy setting with the PVAC regarding the number of applications needed to create (PVI), the number of patients in which (PVI) could be achieved without touch-up applications, the procedure time or the fluoroscopy time.
\end{abstract}

\section{Introduction}

Catheter based pulmonary vein isolation (PVI), is the recommended therapy for drug-refractory atrial fibrillation (AF) [1]. The procedure may be technically challenging, can be time consuming and highly dependent on operators skill. In order to improve the efficacy of PV isolation, shorten the procedure time and learning curve of operators; new specially designed catheters for pulmonary vein isolation have been developed [2-4]. One of these catheters is the Pulmonary Vein Ablation Catheter (PVAC) (Medtronic; Minneapolis, USA), which is a 10-pole circular, over the wire catheter used in combination with a multi-channel, duty-cycled radiofrequency generator (GENius; Medtronic) [2]. The feasibility of the PVAC has been demonstrated in a number of studies and randomized clinical studies have reported similar clinical results compared to point-by-point ablation around the pulmonary veins [2,5-9]. The energy can be delivered in a unipolar or bipolar setting or combined in various ratios. A higher proportion of unipolar energy will give deeper lesions but less energy between the poles possibly leading to non-continuous lines [12]. Although certain energy settings have been recommended for PV isolation there are no randomized studies that have compared the different settings for this purpose. The aim of this study was therefore to compare the efficacy and safety of $\mathrm{PV}$ isolation using a 4:1 versus a 2:1 unipolar/bipolar energy setting with the PVAC. Our hypothesis was that the 2:1 setting, delivering more unipolar energy, would result in deeper and more transmural lesions for pulmonary vein isolation leading to fewer applications and thus shorter procedure times.

\section{Methods}

\section{Patients}

The patients included in this single-center study was a subset of patients in a larger randomized study comparing the PVAC and the CryoCath cryo-balloon (both Medtronic; Minneapolis, MN, USA) and were included between May 2010 and December 2011 [10]. Patients could be included if they were scheduled for a first $\mathrm{PV}$ ablation because of symptomatic paroxysmal or persistent AF resistant to at least one antiarrhythmic drugs. Exclusion criteria were long-standing persistent $\mathrm{AF}$, prior ablation or surgery for $\mathrm{AF}$, congestive heart failure New York Heart Association > III, leftventricular ejection fraction $\leq 30 \%$, left atrial diameter $>6 \mathrm{~cm}$ or left 
atrial thrombus. After signing informed consent patients allocated to the PVAC arm were further randomized to bipolar or unipolar ratio 4:1 or $2: 1$.

All patients were on vitamin $\mathrm{K}$ antagonists with INR $\geq 2$ for at least 4 weeks prior to the ablation. The vitamin $\mathrm{K}$ antagonist was stopped one day before admission and the patients were bridged with dalteparin and heparin to the procedure. A transesophageal echocardiography was performed in all patients 1-2 days prior to the ablation as well as a CT scan of the heart. A three-dimensional reconstruction of the left atrium and the pulmonary vein anatomy was constructed using the CARTO MERGE software (Biosense Webster Inc., Diamond Bar, CA, USA).

\section{Ablation}

All procedures were performed with fully conscious patients given ketobemidon intravenously as analgesics. After vascular access via the right femoral vein, a bipolar catheter was placed in the right ventricular apex, a deca polar catheter in the coronary sinus and an 8-Fr sheath (Daig SL1, St. Jude Medical) was advanced into the left atrium after a transseptal puncture. Anticoagulation using intravenous heparin (loading dose $100 \mathrm{IU} / \mathrm{kg}$ ) was administered and additional heparin was given to achieve an activated clotting time (ACT) of 250-350 seconds throughout the procedure. Thereafter, venograms of the left superior PV and right superior PV were performed. The SL0 sheath was then replaced by a steerable 9 Fr sheath (Channel, Bard Electrophysiology, Lowell, MA, USA) or $10 \mathrm{Fr}$ sheath (CryoCath, Medtronic) allowing the PVAC to be advanced into the left atrium over a 0.032 -inch guide wire. The PVAC was positioned at the antrum of each PV by placing the guide-wire in PV branches to achieve good support. Energy delivery is controlled by a multi-channel, dutycycled radiofrequency generator (GENius; Medtronic). The generator has five preset energy settings: all bipolar, all unipolar and three ratios (4:1, 2:1 and 1:1) of bipolar-to-unipolar energy delivery when the system automatically switches between the two. The recommended settings, by the catheter producer, for performing PV isolation are either 4:1 or 2:1 ratio. Ablation was performed for 60 seconds, set to bipolar/unipolar RF energy ratio, either $4: 1$ or $2: 1$, according to the randomization, targeting $60^{\circ} \mathrm{C}$. Each electrode is supplied with a thermocouple allowing continuous individual temperature monitoring. Maximum power is limited to $8 \mathrm{~W}$ per pole in the $4: 1$ setting and to $10 \mathrm{~W}$ in the 2:1 setting. The five bipolar channels can be activated individually. All poles that were considered to have good wall-contact, based on the local electrogram amplitude, in the antrum were activated. A temperature that did not reach $50^{\circ} \mathrm{C}$, was regarded as an indication of poor wall contact with that electrode-pair, and was therefore turned off during the application. The PVAC was rotated or adjusted when needed to target areas of the antrum not covered previously or to sites with the earliest PV potentials. Pulmonary vein conduction block was confirmed by mapping with a circular catheter (Lasso, Biosense Webster) during sinus rhythm and atrial pacing. In case of remaining $\mathrm{PV}$ potentials additional applications were applied with the PVAC with the previous RF energy setting. In case the encircling could not be completed around a vein, change to a conventional RF ablation catheter was allowed.

The procedure time, the number of applications as well as the total application time for completing PV isolation, the number of channels activated during each application and the fluoroscopy time were recorded.

\section{Follow-up}

All patients continued on oral anticoagulation for at least 4 weeks after the procedure and in case of risk factors for thromboembolic complications they continued on anticoagulation throughout the study. The antiarrhythmic drug treatment used at the time of ablation was continued to the 3-month follow-up and then stopped if there were no symptoms of AF. At 6 month all patients underwent a clinical investigation including a CT-scan and a transthoracic echocardiography.

The study complies with the Declaration of Helsinki and the Regional Ethics Review Board approved the research protocol. All patients gave written informed consent to participate in the study.

\section{Statistical analysis}

Continuous variables are expressed as mean \pm SD. Comparisons between groups were made by Fischer's exact test for categorical variables and with Mann-Whitney's test for continuous variables. A $\mathrm{P}$-value $<0.05$ was considered statistically significant.

\section{Results}

Thirty-five patients with a mean age of $62 \pm 7.7$ years (range 41-75 years) were included in the study. The number of women in the population was 12 representing 34\%. In the population, 54\% presented with paroxysmal AF at baseline. The history of AF varied widely, ranging between one and 34 year, mean 8.4 years. Baseline demographics of each group are summarized in table 1 . The only variable that differed between the groups was arterial hypertension that was more frequent in the group randomized to RF 4:1.

\section{Procedural results}

The mean number of applications with the PVAC catheter to isolate all veins or before switching to another catheter was $41 \pm 10$ and $51 \pm 15(p=0.3)$ respectively with the $4: 1$ and 2:1 setting. The number of successful pulmonary vein isolations, with the 4:1 and 2:1 setting respectively, is shown in table 2 . A switch to a conventional RF ablation catheter was required in 3 patients with the $4: 1$ setting

Table 1: Baseline demographics.

\begin{tabular}{|l|c|c|}
\hline & RF 4:1 group & RF 2:1 group \\
\hline No of patients & 17 & 18 \\
\hline Female/male (n) & $5 / 12$ & $7 / 11$ \\
\hline Age (years) & $61 \pm 8$ & $63 \pm 8$ \\
\hline AF duration (years) & $10 \pm 10$ & $7 \pm 6$ \\
\hline Paroxysmal AF/persistent AF (n,\%) & $7 / 10(41 / 59 \%)$ & $12 / 6(67 / 33 \%)$ \\
\hline Hypertension (n,\%) & $12(71 \%)$ & $10(56 \%)$ \\
\hline Diabetes (n, \%) & $1(6 \%)$ & $1(6 \%)$ \\
\hline Coronary artery disease $(n, \%)$ & $2(12 \%)$ & $2(11 \%)$ \\
\hline Left ventricular dysfunction $(n, \%)$ & 0 & 0 \\
\hline Body mass index & $27 \pm 3$ & $27 \pm 3$ \\
\hline
\end{tabular}


Table 2: The number of vein that could be isolated with PVAC alone, mean electrode pairs activated, total RF time and fluoroscopy time with the different RF settings.

\begin{tabular}{|l|c|c|c|}
\hline \multicolumn{1}{|c|}{} & \multicolumn{2}{|c|}{ Energy setting } & \\
\hline Isolated veins $(\mathrm{n}, \%)$ & & $\mathbf{2 : 1}$ & p value \\
\hline LSPV & $15 / 17(88)$ & $16 / 18(89)$ & $\mathrm{NS}$ \\
\hline LIPV & $15 / 17(88)$ & $14 / 18(78)$ & $\mathrm{NS}$ \\
\hline RSPV & $15 / 17(88)$ & $17 / 18(94)$ & NS \\
\hline RIPV & $15 / 17(88)$ & $12 / 18(64)$ & NS \\
\hline Electrode pairs used (mean, SD) & $2.21 \pm 0.33$ & $1.87 \pm 0.38$ & NS \\
\hline Total RF time, min (mean \pm SD) & $40.8 \pm 12$ & $44.3 \pm 13$ & NS \\
\hline Flouroscopy time, min (mean \pm SD) & $42 \pm 14$ & $50 \pm 17$ & NS \\
\hline
\end{tabular}

LSPV = left superior pulmonary vein; LIPV = left inferior pulmonary vein; RSPV = right superior pulmonary vein; RIPV = right inferior pulmonary vein. $\mathrm{RF}=$ radiofrequency. In the 6 cases with common ostium left a success-ful/unsuccessful isolation is presented as successful/unsuccessful for both LSPV and LIPV

and in 7 patients with 2:1 setting. Complete PV isolation of all veins could be achieved in 16 of 17 patients in the 4:1 group as compared with 16 of 18 patients in the 2:1 group. The reasons for unsuccessful PV isolation were a large left common ostium in one patient that could not be isolated despite many applications, presumably due to oedema formation. One patient had intolerable pain whenever RF ablation was initiated late in the procedure and the procedure was therefore stopped. In another patient, introduction of a second sheet was planned but could not be managed due to problems with vessel access and subsequent hematoma.

One patient in each group had also isthmus-dependent atrial flutter and an isthmus ablation in the right atrium was also performed. The total procedure time and fluoroscopy time includes these procedures as well. The procedure time from venous access to withdrawal of all sheets was $155 \pm 35$ minutes with the $4: 1$ setting and $174 \pm 41$ minutes with the $2: 1$ setting (n.s.). The total radiofrequency application time and the mean number of RF-electrode pairs that was active during the applications is shown in table 2 . The total fluoroscopy time, including the time for touch-up with another catheter, was $42 \pm 14$ with the 4:1 mode and $50 \pm 17$ with 2:1 mode (n.s.).

There were no major complications during the procedure such as: death, tamponade, TIA/stroke or phrenic nerve palsy.

\section{Follow-up}

At six-month follow-up no patients had experienced any late complications and no pulmonary vein stenosis was observed at CT scan. The result of the main study regarding freedom from AF has been published elsewhere [10] and the populations in this study are not powered to detect differences between clinical outcome measures such as freedom from AF.

\section{Discussion}

The main finding in this study is that there was no statistically significant difference between the 4:1 and 2:1 bipolar-to-unipolar energy setting with the PVAC regarding the number of applications needed to create pulmonary vein isolation. There was further no difference in the number of patients in which pulmonary vein isolation could be achieved without touch-up applications, the procedure time or the fluoroscopy time between the two energy settings. If anything, there was a non-significant trend for shorter procedures with fewer applications and shorter fluoroscopy times with the 4:1 setting than with the 2:1 setting, which was the contrary to our initial hypothesis. The reason could be that the $4: 1$ setting, giving relatively more energy in bipolar mode, creates more continuous lines than the 2:1 setting. The 2:1 setting gives more energy in unipolar mode which creates deeper lesions but with the possible risk of gaps between the poles. Several studies have reported a gradual increase in lesion depth by increasing the ratio of unipolar energy delivered by the PVAC [1112]. Wijffels et al. [11] reported that all energy setting (unipolar, 1:1, 2:1 and 4:1 bipolar/unipolar ratio) produces transmural lesions. This was, however, studied in pigs with radiofrequency applications in the superior caval vein, which anatomically does not resemble the human left atrium. Presumably applications with the $4: 1$ setting gives transmural lesions in the human heart as well, and increasing the lesion depth by ablating with the 2:1 setting would not add any clinical benefit. Wieczorek et al. [13] has recently published a randomized study between the 4:1 versus 2:1 ablation mode with the PVAC, in patients undergoing a redo procedure, in which the 2:1 mode resulted in shorter procedure time and shorter fluoroscopy time. This was due to less risk of reconnection within a 30 minute waiting time in the 2:1 group, indicating that the deeper lesion created by the 2:1 setting is needed in some areas. We did not have a waiting time in this study since that was not clinical routine at the time the study was planned. Another possible reason that we did not see any benefit with the 2:1 setting could be that this setting was more painful and that fewer pairs could be activated at each application. Pain was by far the most common cause for not giving a full application of $60 \mathrm{~s}$, which was more often the case with the 2:1 setting than 4:1 setting. Often, no more than one to three electrode-pairs could be activated at each application despite electrogram reflecting good wall contact with the 2:1 setting. This necessitates more applications to create PV isolation, the risk of non-continuous lines and prolongation of the procedure. If the procedure had been performed under general anesthesia, pain had not been a limiting factor, which may have affected the results. In the study by Wieczorek et al. [12], all procedures were performed under general anesthesia or deep sedation. Our clinical routine for all types of catheter ablations has always been to perform the procedure with the patient awake because we believe this decrease risk for complications.

We did not have any major complications during the procedure or during the first 6 months follow-up. The study is, however, not powered to rule out any differences in complication rates between the two settings.

A limitation to this study is the small study population which may disguise minor differences between the groups. The results, however, points to a trend towards the 4:1 energy setting being superior to the 2:1 setting thus being contrary to our hypothesis that the $2: 1$ setting would be superior.

\section{References}

1. Camm AJ, Lip GY, De Caterina R, Savelieva I, Atar D, et al. (2012) 2012 
focused update of the ESC Guidelines for the management of atria fibrillation: an update of the 2010 ESC Guidelines for the management of atrial fibrillation--developed with the special contribution of the European Heart Rhythm Association. Europace 14: 1385-413.

2. Boersma LV, Wijffels MC, Oral H, Wever EF, Morady F (2008) Pulmonary vein isolation by duty-cycled bipolar and unipolar radiofrequency energy with a multielectrode ablation catheter. Heart Rhythm 5: 1635-1666.

3. Schmidt B, Antz M, Ernst S, Ouyang F, Falk P, et al. (2007) Pulmonary vein isolation by high-intensity focused ultrasound: first-in-man study with a steerable ballon catheter. Heart Rhythm 4: 575-584.

4. Neumann T, Vogt J, Schumacher B, Dorszewski A, Kuniss M, et al. (2008) Circumferential pulmonary vein isolation with the cryoballoon technique results from a prospective 3-center study. J Am Coll Cardiol 52: 273-278.

5. Mulder AA, Wijffels MC, Wever EF, Boersma LV (2012) Freedom from paroxysmal atrial fibrillation after successful pulmonary vein isolation with pulmonary vein ablation catheter-phased radiofrequency energy: 2-year follow-up and predictors of failure. Europace 14: 818-825

6. Beukema RP, Beukema WP, Smit JJ, Ramdat Misier AR, Delnoij PP, et al (2010) Efficacy of multi-electrode duty-cycled radiofrequency ablation for pulmonary vein disconnection in patients with paroxysmal and persistent atrial fibrillation. Europace 12: 502-507.

7. Mulder AA, Balt JC, Wijffels MC, Wever EF, Boersma LV (2012) Safety of pulmonary vein isolation and left atrial complex fractionated atrial electrogram ablation for atrial fibrillation with phaser radiofrequency energy and multielectrode catheters. Europace 14: 1433-1440.
8. Bittner A, Monnig G, Zellerhoff S, Pott C, Kobe J, et al. (2011) Randomized study comparing duty-cycled bipolar and unipolar radiofrequency with pointby-point ablation in pulmonary vein isolation. Heart Rhythm 8: 1383-1390.

9. Bulava A, Hanis J, Sitek D, Osmera O, Karpianus D, et al. (2010) Catheter ablation for paroxysmal atrial fibrillation: a randomized comparison between multielectrode catheter and point-by-point ablation. Pacing Clin Electrophysiol 33: $1039-1046$

10. Malmborg H, Lönnerholm S, Blomström P, Blomström -Lundqvist C (2013) Ablation of atrial fibrillation with cryoballoon or duty-cycled radiofrequency pulmonary vein ablation catheter: a randomized controlled study comparing the clinical outcome and safety; the AF-COR study. Europace 15: 1567-1573.

11. Wiffels MCEF, Van Oosterhout M, Boersma LVA, Werneth R, Kunis C, et al. (2009) Characterization of in vitro and in vivo lesions made by a novel multichannel ablation generator and a cicumlinear decapolar ablation catheter. J Cardiovasc Electrophysiol 20: 1142-1148.

12. Lau M, Hu B, Werneth R, Sherman M, Oral H, et al. (2010) A theoretical and experimental analysis of radiofrequency ablation with a multielectrode, phased, duty-cycled system. PACE 33: 1089-1100.

13. Wieczorek M, Hoeltgen R, Tajtaraghi S, Lawrenz W, Lukat M (2013) Pulmonary vein re-isolation for atrial fibrillation using a duty-cycle phased radiofrequency ablation: safety and efficacy of a primary 2:1 bipolar/unipolar ablation mode. J Interv Card Electrophysiol 36: 55-60.

Copyright: (C) 2014 Lönnerholm S, et al. This is an open-access article distributed under the terms of the Creative Commons Attribution License, which permits unrestricted use, distribution, and reproduction in any medium, provided the original author and source are credited. 